\title{
WORLD-WIDE AERONAUTKCAL SATELLTE COMMUNICATIONS
}

\author{
Peter Wood, Manager-Aeronautical Department, INMARSAT \\ Keith Smith, Project Leader-Aeronautical Department, INMARSAT \\ INMARSAT \\ 40 Melton Street \\ London, NW1 2EQ \\ England.
}

\section{ABSTRACT}

Faced with the resurgence of interest in aeronautical satellite communications, and with the encouragement of aviation organizations and its Signatories, INMARSAT decided to expand the spectrum covered by its new generation of satellites, INMARSAT-2, to include $1 \mathrm{MHz}$ (subsequently increased to $3 \mathrm{MHz}$ ) of the spectrum designated for aeronautical use. It began a design study which led to the specifications for the system that is now being implemented. Subsequently INMARSAT has awarded contracts for the design of avionics and high gain antennas to a number of manufacturers, while several of the Signatories that provide ground equipment for communicating with the INMARSAT satellites are modifying their earth stations to work with the avionic equipment. As a result of these activities, a world-wide aeronautical satellite system supporting both voice and data will become operational in 1989.

\section{SERVICE REQUIREMENTS.}

There are four generic applications that will benefit from the use of satellite communications:

- air trafíc services

communications for air traffic control purposes, including automatically relaying the position of an aircraft as determined by the on-board navigation equipment to the air traffic control center (automatic dependent surveillance)

- airline operational control communications which involve the regularity and efficiency of flights, such as between an aircraft and its operating airline giving its estimated time of arrival.

- airline administrative communications non-safety related communications which affect the administration of an airline. An example would be passenger connecting flight arrangements.

- passenger correspondence communications to and trom a passenger in flight. These would normally relate to telephone services, but could include telex and facsimile.

To meet the needs of these services, three basic forms of communication are required:

- a low data rate service ( $600 \mathrm{bit} / \mathrm{sec}$ ). This has been defined by ICAO's FANS committee as the "core" system.

- a medium data rate service (up to $12.6 \mathrm{~kb} / \mathrm{s}$ ), and

- a voice service, with a quality equivalent to that of a good telephone service ("toll" quality). At a later date, other services (such as a lower quality voice channel) may be necessary. 


\section{SYSTEM ELEMENTS}

The major elements of the aeronautical satellite system comprise:

(a) the Space Segment, in particular the satellite communications transponders, and the associated frequency bands assigned for use by the aeronautical satellite system. Figure 1 shows the provisions of INMARSAT-2 satellites compared with the outcome of Mobile-WARC-87;

(b) Aircraft Earth Stations (AES) which interface in the aircraft with ACARS/AIRCOM and other data equipment, and with crew and passenger voice equipment, and with the space segment at L-band;

(c) Aeronautical Ground Earth Stations (GES)* which interface with the space segment at Cand L-bands, and with terrestrial voice and data networks;

(d) Network Coordination Stations (NCS), which interface with the GESs for the purpose of allocating satellite capacity.

INMARSATs present space segment comprises nine satellites, three in each ocean region. One satellite in each region is classified as the "operational" satellite, the other two as spares. The three operational satellites provide effectively global coverage and INMARSAT reconfigures the satellite disposition from time to time to match traffic demand. These first generation satellites will be augmented from late 1989 by at least 3 2nd generation satellites (INMARSAT-2) which like the first generation, each offer "global beam" coverage. Figure 2 shows the coverage at 0 and 5 degree elevation angles. Plans are well under way for the introduction of spot-beam INMARSAT satellites in the 1991/93 timeframe.

The major L-band parameters of the first and second generation INMARSAT satellites are (at edge of coverage):

\begin{tabular}{|c|c|c|}
\hline $\begin{array}{l}\text { Satellite/ } \\
\text { Package }\end{array}$ & $\begin{array}{l}\text { EIRP } \\
\text { (dBW) }\end{array}$ & $\begin{array}{l}G / T \\
(d B / K)\end{array}$ \\
\hline Marecs & 34.5 & -11.2 \\
\hline MCS & 33.0 & -13.0 \\
\hline Marisat & 27.0 & -17.0 \\
\hline INMARSAT-2 & 39.0 & -12.5 \\
\hline
\end{tabular}

\section{SYSTEM DESIGN CONSIDERATIONS}

The system design uses a hybrid FDMATDMATDM approach. Data and signalling use a TDM/TDMA packet-transmission technique, while digital voice circuits use single channel per carrier (SCPC) transmission in order to take advantage of the characteristics of speech to minimize satellite resource demands. The factors that were considered included:

- The need to provide a global capability from the start of service.

- Spectral efficiency

- Power conservation

Many system designs were considered, but the reasons for choice of an SCPC approach to voice transmission appear from the analysis summarised in Table 1.

- Ground Earth stations are generally owned and operated by Signatories --organizations nominated by their countries to invest in, and work with, INMARSAT. The Signatories are the organizations actually responsible for the provision of aeronautical satellite communication services. 
Table 1 is based upon the SCPC design selected by INMARSAT, and a specific alternative TDMA approach that has been considered by the aeronautical industry. Both of these have been considered by the Airlines Electronic Engineering Committee (AEEC). It is apparent that, whether operating with global or spot beam satellites, or with high gain or low gain antennas,an SCPC system offers more capacity.

Contributory factors are:

- the transmitted power from the satellite is proportional to the number of channels being handled; for a TDMA system the power is constant, irrespective of the number of channels in use at one time,

- the transmitted power can be adjusted on each channel to deal with variations in path length, propagation conditions, and variations in antenna gain. This allows reduction of system margins and consequent power savings. In a TDMA system, the power has to be at a constant worst-case level.

- it is possible to use voice activation on voice circuits, using no power until someone is actually speaking.

- use of SCPC avoids the need to 'bundle' traffic into groups of circuits of preassigned size. Such bundling not only restricts system flexibility, but incurs significant efficiency penalties arising from the statistical nature of voice traffic.

Nevertheless, the present choice of SCPC does not preclude the future development of a more suitable TDMA design following the widespread introduction of spot beam satellites.

\section{SYSTEM CONFIGURATION}

The INMARSAT aeronautical system is configured around two types of transmission channel, data and voice, and two R.F. amplifier/antenna combinations on the aircraft, low-gain $(0 \mathrm{dBi})$ and high gain (12 dBi). Aircraft Earth Stations (AES) are classified according to their equipment configurations and capabilities, as follows:

Class 1: Low gain antenna only, data services only;

Class 2: High gain antenna only, voice services only;

Class 3: High gain antenna only, voice and data services;

In the initial configuration, transmissions over the data channel will be at either 600 or 1200 bits/second (selected by the ground earth station). As rate 1/2 forward error correction (FEC) coding is used to reduce the bit error rate, the effective information data rates are 300 and $600 \mathrm{bits} / \mathrm{second}$ respectively.

Voice signals are digitized at a $9.6 \mathrm{kbits} / \mathrm{s}$ rate, and are combined with signalling data, resulting in an information data rate of $10.5 \mathrm{kbits} / \mathrm{s}$. Again, FEC encoding is used to reduce the bit error rate, giving a data transmission rate of $21 \mathrm{kbits} / \mathrm{s}$ over the voice channel.

The specific service capabilities of an AES will vary according to its class and the number of channel units installed. Examples of typical installations would be:

(a) one data channel only. This would support data services only, to a single GES;

(b) one data channel, plus two voice channels. This would support data and voice services simultaneously, with the voice and data able to use different GESs; and

(c) two data channels, plus six voice channels. The data services could be to two different GESs. The voice services could use several GESs.

\section{SYSTEM DESIGN SUMMARY}

\section{Modulation and Coding}

Up to an RF transmission rate of 2400 bits, binary modulation is used with root $40 \%$ raised cosine filtering, and above that a quadrature technique is used with root $60 \%$ raised cosine filtering. 
Both are "offset" techniques which minimize RF amplitude variations after filtering, and thereby permit close spacing of the RF channels. For the quadrature technique, initial channel spacings of about 0.83 of the bit rate are achieved.

FEC coding is provided, with rates of $1 / 2,3 / 4$ and 1 (no coding). Constraint-length seven convolutional coding is used, and interleaving is applied to randomize the bursty errors arising from the multipath L-band channel, which has a fade rate of about $30 \mathrm{~Hz}$ and above, with $\mathrm{C} / \mathrm{M}$ of $10 \mathrm{~dB}$ and above. A common interleaver structure is applied at all bit rates, although the block size is varied to maintain a constant depth (randomizing power) to match the bit rate. When FEC-coded channels are used for voice transmission, the maximum interleaving delay is kept to between 30 and $40 \mathrm{mS}$, which slightly reduces the effectiveness of interleaving. It is also possible to operate without FEC. Transmission Format (Data Link Layer)

Given the nature of the multipath fading channel, it is vital to design the transmission format to ensure the maximum resistance to burst errors. This implies a need for consistent link-level error detection performance, and the availability of error recovery procedures.

This has been handled at link layer by formatting all data, whether signalling, control, or user-data, into standard packets of pre-defined length, each protected by a common CCITT cyclic redundancy (CRC) code to ensure consistent packet-error detection. The format and length of these "Signal Units" (SUs) has been the subject of considerable study, representing a trade-off between efficiency (link-layer overheads, compatibility with signals and other message lengths) and error performance in the RF channel. The system user may request that packets are delivered to the application without error checking (Direct Link Service) or after ARQ (Reliable Link Service). The standard SU is 96 bits, of which 16 are Cyclic Redundancy Check (CRC) for error detection. Where included, aircraft addresses are 3 octets in length, and ground station addresses are one octet.

Data Applications (Network Layer)

The system provides full support for a network layer consistent with the ISO layered architecture for connectionless, connection-oriented, and special (e.g., polling) services.

Transmission Channel Characteristics

To accommodate the range of services, four basic RF transmission channels have been defined with different satellite access techniques. The four channel types are:

P-Channel:

Packet mode Time Division Multiplex (TDM) channel, used in the forward direction (ground-to-air) to carry signalling and user data. The transmission is continuous from each earth station in the satellite network.

R-Channel:

Random access (slotted Aloha) channel, used in the return direction (aircraft-to-ground) to carry some signalling and user data (short messages), specifically the initial signals of a transaction, typically request signals.

T-Channel:

Reservation Time Division Multiple Access (TDMA) channel, used in the return direction only. The receiving earth station reserves time slots for transmissions requested by aircraft earth stations, according to message length. The sending aircraft earth station transmits the messages in the reserved time slots according to priority.

C-Channel:

Bi-directional circuit-mode channel. The use of the channel is controlled by assignment and release signalling at the start and end of each call. A C-channel has a BER after FEC decoding of better than $10^{-3}$ (whereas the $P, R$ and $T$ channels have better than $10^{-5}$ ) which is suitable for voice communications. Each $C$-channel may carry one or more voice channels, but to support more than two voice channels, more than one RF carrier is needed. A C-channel also carries a multiplexed low-rate data channel (about $200 \mathrm{bit} / \mathrm{s}$ ) for signalling or user data. 


\section{TABLE 1}

TDMAFDMA COMPARISON: NUMBER OF AIR TO GROUND VOICE CHANNELS PER AIRCRAFT AS A FUNCTION OF HPA POWER

\begin{tabular}{|c|c|c|c|c|c|c|}
\hline AIRCAAFT ANTENNA GAIN (SAT. ELEVW.) & \multicolumn{2}{|c|}{12 dBic (> 200) } & \multicolumn{2}{|c|}{12 aBiC (> 5) } & \multicolumn{2}{|c|}{0 dBic $(>200)$} \\
\hline SPACECAAFT ANTENNA GAIN (EOC) & \multicolumn{2}{|c|}{ Glober (17 dBic) } & \multicolumn{2}{|c|}{ Spor (25 OABC) } & \multicolumn{2}{|c|}{ Spot (25 dBic) } \\
\hline MPA POWER & FDMA & TOMM & FDMA & Toma & Fom & TOMA \\
\hline $40 W$ (AEEC) & 4 & 0 & 14 & 6 & 2 & 0 \\
\hline $30 \mathrm{~W}$ & 3 & 0 & 10 & 6 & 1 & 0 \\
\hline $20 \mathrm{~W}$ & 2 & 0 & 7 & $6 *$ & 1 & 0 \\
\hline $12 W$ & 1 & $\mathbf{0}$ & 4 & 0 & 0 & 0 \\
\hline $6 \mathrm{~W}$ & 0 & $\mathbf{0}$. & 2 & 0 & 0 & 0 \\
\hline $3 w$ & 0 & $\mathbf{0}$ & 1 & 0 & 0 & 0 \\
\hline
\end{tabular}

(Class-C) may be used for single-carier cases

TDMA/FDMA COMPARISON: NUMBER OF GROUND TO AIR VOICE CHANNELS PER SATELLITE SPOT BEAM

\begin{tabular}{|c|c|c|c|c|}
\hline Aircratt Amtenna Gain & & & & \\
\hline \multirow{2}{*}{$\begin{array}{l}\text { Satellite Beam } \\
\text { EIRP (dBW, eoc) }\end{array}$} & \multicolumn{2}{|c|}{ Number of voice channels } & \multicolumn{2}{|c|}{ Number of voice channels } \\
\hline & FOMA & TOMA & FDMM -.. & TOMn \\
\hline 36 & 100 & - & 4 & 0 \\
\hline $39 *$ & 200 & 30 & 9 & 0 \\
\hline 42 & 400 & $30+30^{\circ}$ & 20 & $\mathbf{0}$ \\
\hline
\end{tabular}

- Requires 2 transponders

-.. Rechuced voice activation ectvantege

- AvSat nominal (approx.)

System Deelgns: TOMA: as per ARINC Ouick Check 39 (AvSet System Technical Description - 8.0 kbit/s voice)

FDMA: es per INMARSAT System Definition Manual - 9.6 kbit/s voloe

eac: edge of coverage $=5^{\circ}$ elevation 
FIGURE 1 - L-BAND FREQUENCY ALLOCATION

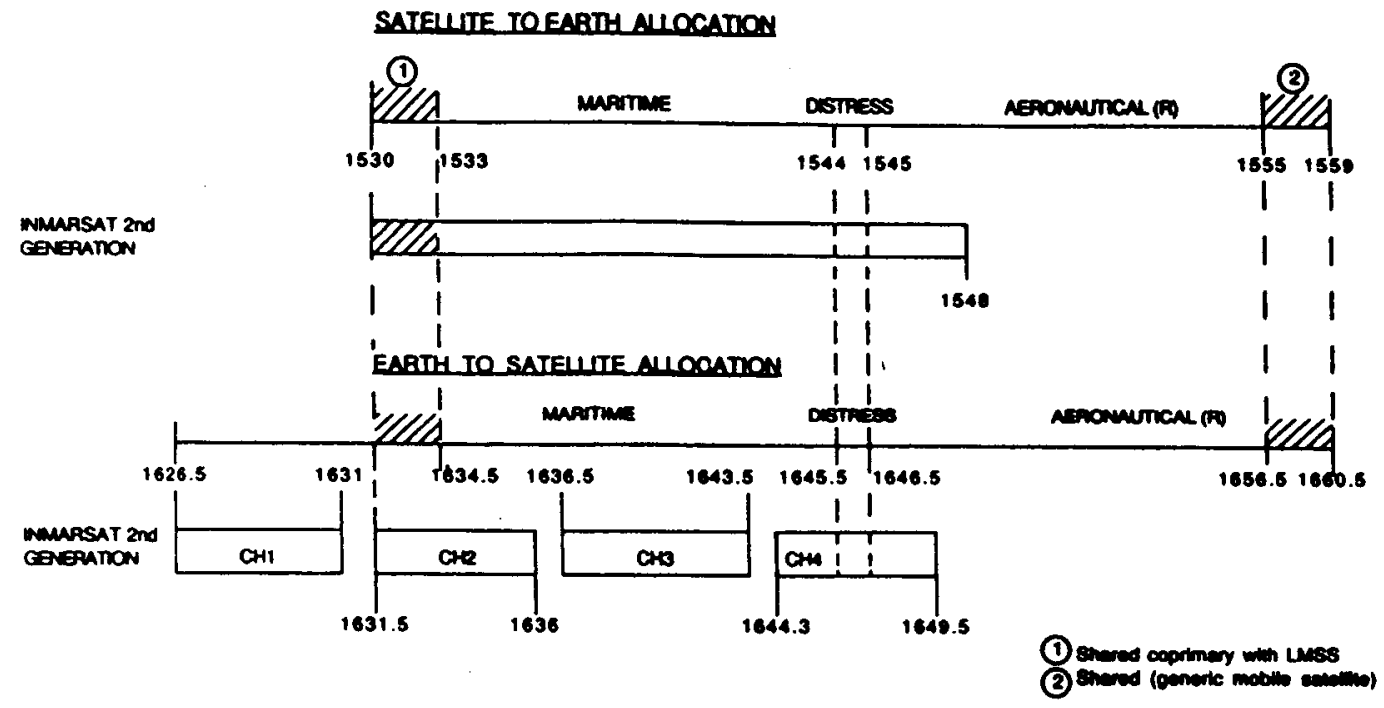

FIGURE 2 - INMARSAT GLOBAL COVERAGE

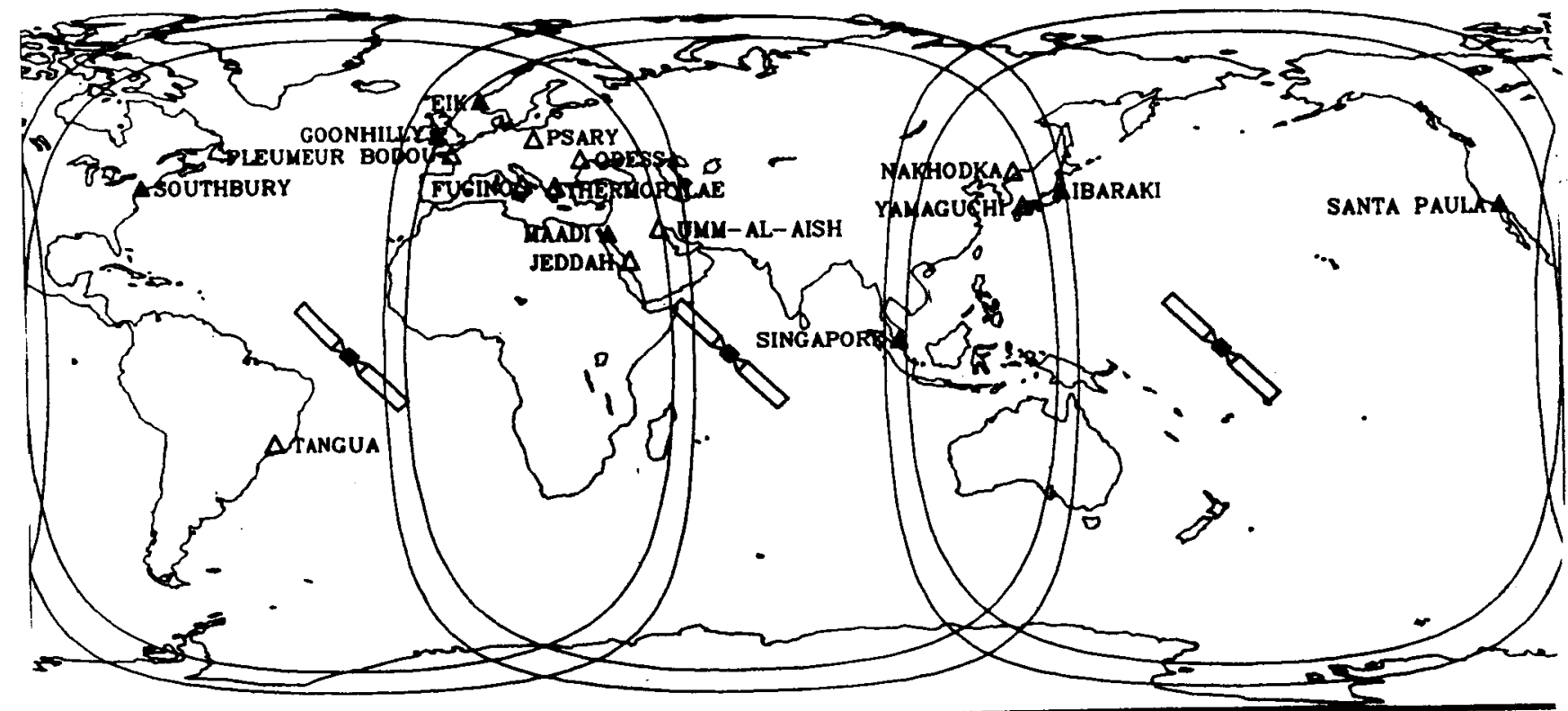

Satellite locations:

AERONAUTICAL GROUND EARTH STATIONS PLANNED A AOR 26․ IOR $63^{\circ} \mathrm{E}$, POR $180^{\circ} \mathrm{E}$ MARITIME COAST EARTH STATIONS $\triangle$ 